\title{
Commentary
}

\section{Potential for Underestimation of d-Methylphenidate Bioavailability Using Chiral Derivatization/Gas Chromatography}

\author{
Kennerly S. Patrick and Wendy Rodriguez \\ Department of Drug Discovery and Biomedical Sciences, Medical University of South Carolina, Charleston, South Carolina
}

Received March 15, 2019; accepted April 18, 2019

\begin{abstract}
A tenable hypothesis is presented which explains disparities between older oral dl-MPH bioavailability data generated using chiral derivatization-gas chromatography versus more recent findings using chiral liquid chromatography. These disparities persist in current literature. The gas chromatographic methods found that the absolute bioavailability of d-MPH is $23 \%$ and that of I-MPH is $5 \%$ (i.e., $82 \%$ as the active $d$-isomer), while liquid chromatographic methods consistently report that approximately $99 \%$ of circulating MPH is d-MPH. Older methods used perfluoroacylated S-prolyl derivatizing agents which have a history of imprecision due to the susceptibility of the prolyl S-configuration to isomerize to the R-enantiomer. Accordingly, any R-prolyl impurity in the chiral derivatization reagent yields the (R,R,R)-MPH-prolyl diastereomer which, in being related as the opposite enantiomer of (S,S,S)-prolyl-MPH,
\end{abstract}

co-elutes with I-(S,S)-MPH. This results in overestimation of the percent I-MPH at the expense of underestimating d-MPH. Unless compelling reasons exist to justify use of any chiral discriminators, less complex and less costly achiral analysis of plasma MPH appears appropriate for d-MPH quantitation since $99 \%$ exists as d-MPH. However, simultaneous plasma monitoring of d-MPH and I-MPH may be warranted when alterations in first-pass hepatic metabolism by carboxylesterase 1 (CES1) occurs. For example, (a) with transdermal dl-MPH delivery; (b) in cases of concomitant dl-MPH and a CES1 inhibitor, e.g., ethanol, which elevates I-MPH and d-MPH concentrations; (d) in forensic studies of intravenous or intranasal dl-MPH abuse; (e) were dl-MPH to be formulated as a free base sublingual product; or (f) as emerging advances in dl-MPH gene-dose effects warrant isomer correlations.

We reevaluated the extent to which immediate-release (IR) dlmethylphenidate (dl-MPH) is subject to enantioselective presystemic metabolism within the context of the existing biomedical literature. The following discussion is intended to better define the pharmacokinetics (PK) of oral IR-dl-MPH. In addition, in this letter we offer a rationale for considering selection of less-complex, cost-curtailing bioanalytical methods that obviate chromatographic chiral discriminators when monitoring the psychoactive d-methylphenidate (d-MPH) isomer component of this racemate (Patrick et al., 1987; Patrick and Straughn, 2016). Understanding the PK of IR-dl-MPH underpins rational study designs of new modifiedrelease (MR) dl-MPH formulation technologies (at least six unique branded MR-dl-MPH products have been approved in the last 5 years, with a seventh tentatively approved (Drugs@FDA: FDA Approved Drug Products; https://www.accessdata.fda.gov/scripts/cder/daf/); others are in the regulatory pipeline (Patrick et al., 2019). Furthermore, understanding

This work was supported solely through the National Institutes of Health National Institute of Alcohol and Alcoholism [Grant RO1AA016707]; the Medical University of South Carolina's Clinical and Translational Research Center with support from the National Institutes of Health [Grant MO1RR01070-18]; and the National Institutes of Health National Center for Research Resources Southeastern Pre-Doctoral Training in Clinical Research [Grant 1T32 RR023258].

https://doi.org/10.1124/dmd.119.087189.
dl-MPH absorption and disposition provides guidance for bioanalytical methods used in ongoing gene-/dose-effect studies, which are now showing increasing promise in the advancement of personalized attention-deficit/hyperactivity disorder (ADHD) pharmacotherapy (Zhu et al., 2008; Lyauk et al., 2016; Stage et al., 2017, 2019).

IR-dl-MPH typically provides 4 hours of efficacy in the treatment of ADHD. This psychostimulant undergoes extensive oral first-pass metabolism in humans (Chan et al., 1983) (Table 1), monkeys, and rats (Wargin et al., 1983), whereby the absolute bioavailability of the combined methylphenidate (MPH) enantiomers has been reported to be approximately $30 \%, 22 \%$, and $19 \%$ in these species, respectively. These early 1980s determinations used achiral (nonenantiospecific) gas chromatography (GC) methods. Subsequently, an enantiospecific GC approach using chiral MPH derivatization, chlorphentermine as an internal standard, and electron capture detection found that the isomeric composition of the absolute bioavailable fraction following an oral racemic MPH dose exists as 23\% d-MPH (the $R, R$-isomer) and $5 \%$ 1-methylphenidate (1-MPH) (the $S, S$-isomer) in humans (Srinivas et al., 1993); equating to $82 \%$ of total plasma MPH exposure representing the d-MPH enantiomer (Table 1). Similar percentage differences between $\mathrm{d}-\mathrm{MPH}$ and l-MPH plasma exposure have persisted in more contemporary secondary (Srinivas, 2004) and tertiary biomedical literature studies (Thummel et al., 2018).

ABBREVIATIONS: ADHD, attention-deficit/hyperactivity disorder; CES1, carboxylesterase 1; d-MPH, d-methylphenidate; dl-MPH, dl-methylphenidate; GC, gas chromatography; HFP, heptafluorobutyryl-S-prolyl chloride; IR, immediate release; LC, liquid chromatography; I-MPH, Imethylphenidate; MPH, methylphenidate; MR, modified release; MS, mass spectrometry; PK, pharmacokinetics. 


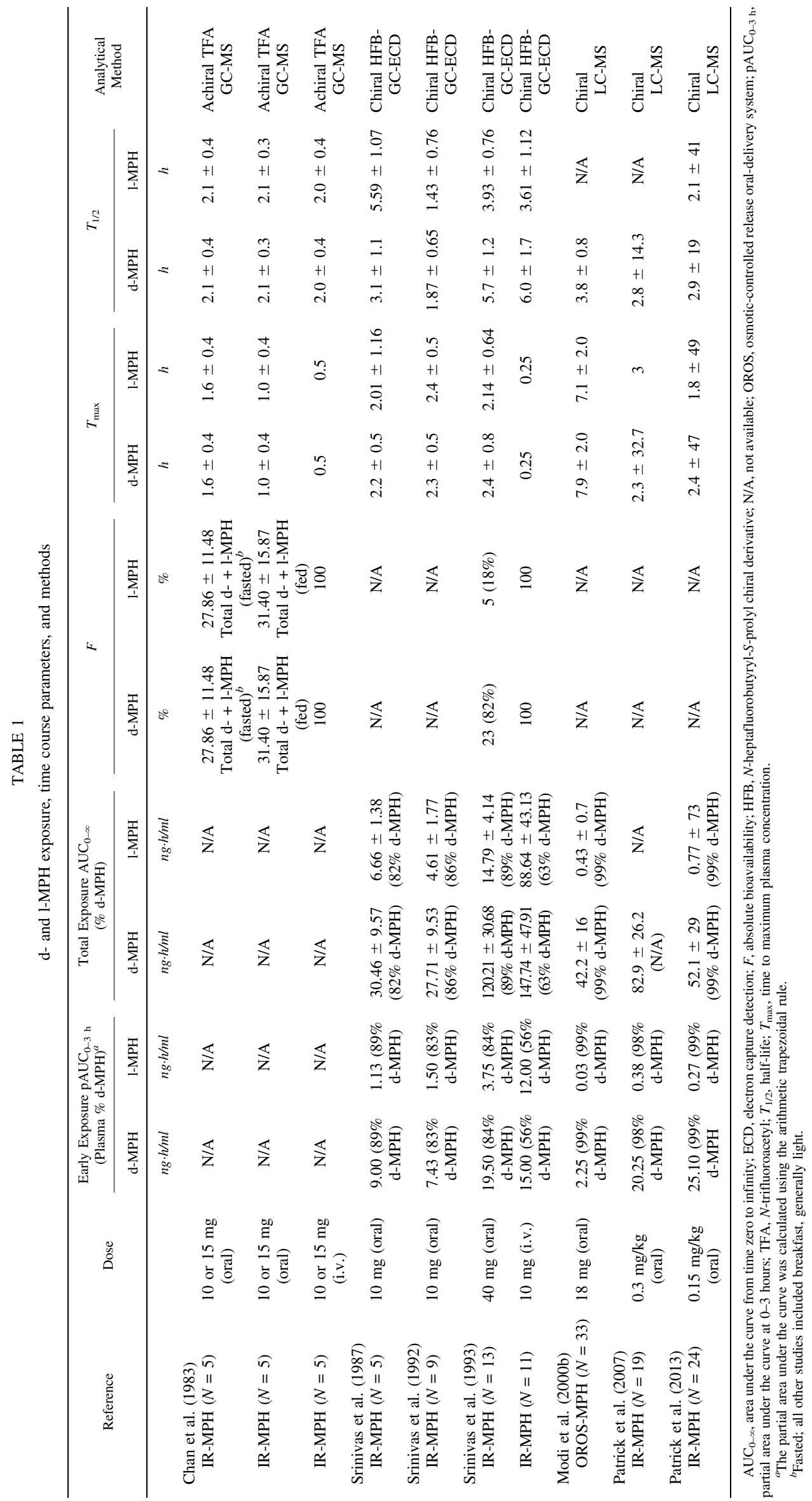

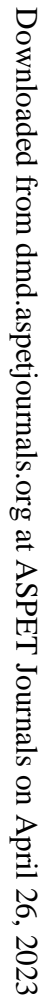


This reported PK relationship between the d:l percentages of plasma MPH isomers notwithstanding, more recent relative bioavailability studies (i.e., where no comparative intravenous dl-MPH PK studies were conducted) have consistently shown that approximately $99 \%$ of total dl-MPH exposure is attributable to the d-MPH isomer. This was revealed by Ramos et al. (1999) in the course of validating a vancomycin-based chiral liquid chromatography (LC)/mass spectrometry (MS) method using IR-dl-MPH in a test subject prior to conducting bioavailability studies of a new MR-dl-MPH product (Lee et al., 2003). This determination of approximate $99 \%$ of oral dl-MPH reaching the systemic circulation has since been replicated in multiple studies of both IR-dl-MPH (Modi et al., 2000a; Patrick et al., 2007, 2013) (Table 1) and MR-dl-MPH formulations (e.g., Modi et al., 2000b). We note that 99\%: $1 \%$ d-MPH:1-MPH systemic exposure following oral dl-MPH could be viewed in practical terms as representing presystemic biocatalytic resolution of racemic dl-MPH to the racemic switch drug: pure d-isomer dexmethylphenidate. Indeed, the package labeling for dexmethylphenidate recommends using one-half the milligram dose when converting a maintenance dose of dl-MPH in an ADHD patient to the enantiopure d-MPH product (Drugs@FDA: FDA Approved Drug Products; https://www.accessdata.fda.gov/scripts/cder/daf/).

More recent bioanalytical studies characterizing enantiospecific plasma MPH concentrations have used chiral LC stationary phases rather than the GC method for MPH enantiomeric separations. These LC methods avoid problematic chiral derivatization (as discussed subsequently), provide the molecular specificity of tandem MS to suppress chemical noise, and incorporate deuterated internal standards that offer optimal analytical control of potential: 1) variability in extraction recovery, 2) extent of postsampling hydrolysis (Ramos et al., 1999), and 3) changes in intraday instrumental performance. Advantages of such an LC-MS analytical approach are evident from published representative chromatograms from LC-MS (Zhu et al., 2011) compared with chiral derivatization GC studies where chemical interferences and loss of baseline resolution are clearly evident (Srinivas et al., 1987).

Chiral derivatization GC methods for PK studies of dl-MPH have most often used heptafluorobutyryl- $S$-prolyl chloride (HFP) to form the corresponding $(S, R, R)$ - and $(S, S, S)$-HFP-MPH diastereomers from $\mathrm{d}-(R, R)-\mathrm{MPH}$ and 1-(S,S)-MPH. The commercial availability of HFP, as used by Lin et al. (1999) for the chiral derivatization of dl-MPH extracted from human plasma, ended many years ago due to the unacceptable degree of in situ racemization during chemical synthesis (enantiomeric excess $<80 \%$; technical support personal communication, January 2019; Campbell Supply Co., Rockton, IL).

The strong electron-withdrawing inductive effects of the seven fluorine bonds on the acyl group of HFP, taken together with the prolyl moiety existing as its acid chloride, can be expected to render the $\alpha$-hydrogen atom especially acidic (Sykes, 1986), and accordingly predispose the stereogenic center to inversion of configuration to the unnatural amino acid $R$-proline antipode. Serving as a precedent, even when using the less fluorinated homolog $N$-trifluoroacetyl-S-prolyl chloride as a chiral GC derivatization reagent required corrections to the relative GC peak areas to establish the enantiomeric excess in the course of preparative scale resolution of dl-MPH isomers. In this example, a reference standard of pure $(S)$-methamphetamine was derivatized with $N$-trifluoroacetyl- $S$ prolyl chloride in parallel with resolution product characterization to adjust for the extent of actual enantiopurity (Patrick et al., 1987). Furthermore, Lui and $\mathrm{Ku}$ (1981) found that commercially available $N$-trifluoroacetyl-S-prolyl chloride contains $5 \%$ to $6 \%$ of the opposite $R$-enantiomer, thus again requiring analogous corrections for enantiospecific GC analysis of a range of amphetamine enantiomeric mixtures.

Any $R$-prolyl impurity in the ( $S$ )-HFP derivatization reagent will yield the $(R, R, R)-\mathrm{MPH}$-prolyl diastereomer, which is the opposite enantiomer of $(S, S, S)$-HFP-MPH and thus coelutes with 1-(S,S)-MPH to pose a likely potential for overestimating the percent of 1-MPH at the expense of underestimating d-MPH. Were this to have been the case, the variance in the late 1980s and early 1990s reports regarding a lesser degree of enantioselective metabolism than now appears to occur finds a compelling scientific basis to explain these conflicting reports of differential isomeric extents of absorption. Thus, it would even appear rational to use less-expensive and less-complex achiral GC or LC methods for plasma d-MPH determinations in view of the vast preponderance of circulating MPH $(\sim 99 \%)$ existing as the active d-MPH enantiomer.

However, simultaneous quantitation of d- and l-MPH is warranted: 1) for transdermal dl-MPH delivery PK, where circumvention of the oral hepatic first-pass effect-the organ that expresses carboxylesterase 1 (CES1), which catalyzes dl-MPH hydrolysis-results in substantial 1-MPH exposure (Patrick et al., 2009); 2) in cases of concomitant dl-MPH and CES1 inhibitor ethanol, where 1-MPH concentrations rise (Patrick at al., 2007, 2013; Zhu et al., 2017); 3) for dl-MPH in combination with other CES1 inhibitors used in clinical practice, e.g., aripiprazole and fluoxetine (Zhu et al., 2010); or 4) in forensic studies of intravenous or intranasal dl-MPH abuse, which again circumvents the hepatic first-pass effect. (Use of intravenous dl-MPH for barbiturate overdose, anesthesia recovery, or intractable hiccups was discontinued in the 1960s.) Were dl-MPH to be formulated as a free base sublingual product, direct oral membrane solubility/transport would again bypass the early hydrolytic effects of hepatic CES1 on dl-MPH (Patrick et al., 2010). It is noted that an orally disintegration tablet (Cotempla XR-ODT) was approved in 2017, which contains dl-MPH not as the hydrochloride salt but rather bound to a nonabsorbable exchange resin. In this formulation, the potential for appreciable oral cavity 1-MPH absorption may warrant investigation.

Also included in Table 1 are the analyses for partial area under the curve at $0-3$ hours. Not only does this time interval cover the primary duration of therapeutic action of IR-dl-MPH, it extends a comprehensive study of partial area under the curve at $0-3$ hours values for a broad range of MR-MPH formulations (Patrick et al., 2019) and carries relevance to this specific partial area under the curve metric in recently instituted bioequivalence parameters now required by the Food and Drug Administration for select dl-MPH products (Jackson, 2014). Taken together with the time to maximum plasma concentration and half-life parameters, Table 1 provides key aspects of both the rate and extent of dl-MPH oral bioavailability.

In summary, a very tenable hypothesis is presented that explains the disparities between older dl-MPH PK data compared with more recent findings, owing to evolving advances in the quality of chiral bioanalytical methodology. As described previously, perfluoroacylated prolyl derivatizing agents have a history of imprecision in enantiospecifically quantifying drugs, including dl-MPH and amphetamines. Currently, dl-MPH serves as a first-line pharmacotherapeutic agent for the treatment of the most commonly diagnosed childhood and adolescent central disorder: ADHD. As empirical approaches to drug individualization give way to pharmacogenomics and precision medicine, fundamental knowledge of well-validated pharmacology is critically important in achieving desired outcomes. This letter serves to correct specific misunderstandings regarding dl-MPH absorption and disposition, which have persisted in mainstream secondary and tertiary biomedical literature (Thummel et al., 2018).

\section{Authorship Contributions}

Performed data analysis: Patrick, Rodriquez.

Wrote or contributed to the writing of the manuscript: Patrick, Rodriquez. 


\section{References}

Chan YP, Swanson JM, Soldin SS, Thiessen JJ, Macleod SM, and Logan W (1983) Methylphenidate hydrochloride given with or before breakfast: II. Effects on plasma concentration of methylphenidate and ritalinic acid. Pediatrics 72:56-59.

Jackson A (2014) Impact of release mechanism on the pharmacokinetic performance of PAUC metrics for three methylphenidate products with complex absorption. Pharm Res 31:173-181.

Lee L, Kepple J, Wang Y, Freestone S, Bakhtiar R, Wang Y, and Hossain M (2003) Bioavailability of modified-release methylphenidate: influence of high-fat breakfast when administered intact and when capsule content sprinkled on applesauce. Biopharm Drug Dispos 24:233-243.

Lin SN, Andrenyak DM, Moody DE, and Foltz RL (1999) Enantioselective gas chromatographynegative ion chemical ionization mass spectrometry for methylphenidate in human plasma. $J$ Anal Toxicol 23:524-530

Lui JH and Ku WW (1981) Determination of enantiomeric $N$-trifuoroacetyl-L-prolyl chloride amphetamine derivatives by capillary gas chromatography/mass spectrometry with chiral and achiral stationary phases. Anal Chem 53:2180-2184.

Lyauk YK, Stage C, Bergmann TK, Ferrero-Milliani L, Bjerre D, Thomsen R, Dalhoff KP, Rasmussen HB, and Jürgens G (2016) Population pharmacokinetics of methylphenidate in healthy adults emphasizing novel and known effects of several carboxylesterase 1 (CESI) variants. Clin Transl Sci 9:337-345.

Modi NB, Lindemulder B, and Gupta SK (2000a) Single- and multiple-dose pharmacokinetics of an oral once-a-day osmotic controlled-release OROS (methylphenidate $\mathrm{HCl}$ ) formulation. $J$ Clin Pharmacol 40:379-388.

Modi NB, Wang B, Noveck RJ, and Gupta SK (2000b) Dose-proportional and stereospecific pharmacokinetics of methylphenidate delivered using an osmotic, controlled-release oral delivery system. J Clin Pharmacol 40:1141-1149.

Patrick KS, Caldwell RW, Ferris RM, and Breese GR (1987) Pharmacology of the enantiomers of threo-methylphenidate. J Pharmacol Exp Ther 241:152-158.

Patrick KS, Radke J, Raymond J, Rodriquez, W, Nguyen L, Koller, L, and Straughn AB (2019) Drug regimen individualization for attention-deficit/hyperactivity disorder: guidance for methylphenidate and dexmethylphenidate formulations. Pharmacotherapy 39:677-688.

Patrick KS, Straughn AB, Minhinnett RR, Yeatts SD, Herrin AE, DeVane CL, Malcolm R, Janis GC, and Markowitz JS (2007) Influence of ethanol and gender on methylphenidate pharmacokinetics and pharmacodynamics. Clin Pharmacol Ther 81:346-353.

Patrick KS, Straughn AB, Perkins JS, and González MA (2009) Evolution of stimulants to treat ADHD: transdermal methylphenidate. Hum Psychopharmacol 24:1-17.

Patrick KS, Straughn AB, Reeves OT III, Bernstein H, Bell GH, Anderson ER, and Malcolm RJ (2013) Differential influences of ethanol on early exposure to racemic methylphenidate compared with dexmethylphenidate in humans. Drug Metab Dispos 41:197-205.

Patrick KS and Straughn AB (2016) Absorption differences between immediate-release dexmethylphenidate and dl-methylphenidate. Drug Met Dispos 44:418-421.

Ramos L, Bakhtiar R, Majumdar T, Hayes M, and Tse F (1999) Liquid chromatography/ atmospheric pressure chemical ionization tandem mass spectrometry enantiomeric separation of dl-threo-methylphenidate, (Ritalin) using a macrocyclic antibiotic as the chiral selector. Rapid Commun Mass Spectrom 13:2054-2062.

Srinivas NR (2004) Clinical pharmacokinetic data of racemic drugs obtained by the indirect method following precolumn diastereomer formation: is the influence of racemization during chiral dervatization significant? Biomed Chromatogr 18:342-349.
Srinivas NR, Hubbard JW, Korchinski ED, and Midha KK (1993) Enantioselective pharmacokinetics of dl-threo-methylphenidate in humans. Pharm Res 10:14-21.

Srinivas NR, Hubbard JW, Quinn D, and Midha KK (1992) Enantioselective pharmacokinetics and pharmacodynamics of $d l$-threo-methylphenidate in children with attention deficit hyperactivity disorder. Clin Pharmacol Ther 52:561-568.

Srinivas NR, Quinn D, Hubbard JW, and Midha KK (1987) Stereoselective disposition of methylphenidate in children with attention-deficit disorder. J Pharmacol Exp Ther 241:300-306.

Stage C, Dalhoff K, Rasmussen HB, Guski LS, Thomsen R, Bjerre D, Ferro-Miliani L, Madsen MB, and Jurgens G (2019) The impact of human CES1 genetic variation on enzyme activity assessed by ritalinic acid/methylphenidate ratios. Basic Clin Pharmacol Toxicol DOI: 10.1111/ bcpt. 13212 [published ahead of print].

Stage C, Jürgens G, Guski LS, Thomsen R, Bjerre D, Ferrero-Miliani L, Lyauk YK, Rasmussen HB, and Dalhoff K; INDICES Consortium (2017) The impact of CES1 genotypes on the pharmacokinetics of methylphenidate in healthy Danish subjects. Br J Clin Pharmacol 83: $1506-1514$.

Sykes P (1986) The strengths of acids and bases, in Mechanisms in Organic Chemistry, 6th ed, pp 59-61, John Wiley \& Sons, Inc., New York.

Thummel KE, Shen DD, and Isoherranen N (2018) Chapter 12: Adrenergic Agonists and Antagonists, Methylphenidate; and Appendix II: design and optimization of dosage regimens: pharmacokinetic data, in Goodman \& Gilman's: The Pharmacological Basis of Therapeutics, 13th ed (Brunton LL, Hilal-Dandan R, and Knollmann BC eds) McGraw-Hill Medical, New York.

Wargin W, Patrick K, Kilts C, Gualtieri CT, Ellington K, Mueller RA, Kraemer G, and Breese GR (1983) Pharmacokinetics of methylphenidate in man, rat and monkey. J Pharmacol Exp Ther 226:382-386.

Zhu HJ, Appel DI, Peterson YK, Wang Z, and Markowitz JS (2010) Identification of selected therapeutic agents as inhibitors of carboxylesterase 1: potential sources of metabolic drug interactions. Toxicology 270:59-65.

Zhu HJ, Patrick KS, and Markowitz JS (2011) Enantiospecific determination of $d l$-methylphenidate and $d l$-ethylphenidate in plasma by liquid chromatography-tandem mass spectrometry: application to human ethanol interactions. J Chromatogr B Analyt Technol Biomed Life Sci 879: 783-788.

Zhu HJ, Patrick KS, Straughn AB, Reeves OT III, Bernstein H, Shi J, Johnson HJ, Knight JM, Smith AT, Malcolm RJ, et al. (2017) Ethanol interactions with dexmethylphenidate and dl-methylphenidate Spheroidal Oral Drug Absorption Systems in healthy volunteers. J Clin Psychopharmacol 37:419-428.

Zhu HJ, Patrick KS, Yuan HJ, Wang JS, Donovan JL, DeVane CL, Malcolm R, Johnson JA, Youngblood GL, Sweet DH, et al. (2008) Two CES1 gene mutations lead to dysfunctional carboxylesterase 1 activity in man: clinical significance and molecular basis. Am J Hum Genet 82:1241-1248.

Address correspondence to: Dr. Kennerly S. Patrick, Department of Drug Discovery and Biomedical Sciences, 280 Calhoun St., QF218; P.O. Box 250140, Charleston, SC 29425. E-mail: patrickk@musc.edu 